\title{
Hipertensão arterial e alcoolismo em trabalhadores de uma refinaria de petróleo
}

\author{
Carlos Tadeu da Silva Lima, ${ }^{1}$ Fernando Martins Carvalho, ${ }^{2}$ \\ Cláudio de Almeida Quadros, ${ }^{3}$ Henrique Ribeiro Gonçalves, ${ }^{3}$ \\ José Américo Seixas Silva Junior, ${ }^{3}$ Maria Fernanda Tourinho \\ Peres $^{3}$ e Mirian Santos Bonfim ${ }^{4}$
}

RESUMO O papel desempenhado pelo padrão de ingesta alcoólica sobre a incidência de hipertensão arte rial ainda não está totalmente esclarecido; além disso, são raros os estudos realizados em popu lações de trabalhadores. Assim, o objetivo do presente trabalho foi avaliar a associação entre al coolismo e hipertensão arterial entre trabalhadores de uma refinaria de petróleo em Mataripe, Estado da Bahia, Brasil, de 1986 a 1993. O estudo foi de coorte retrospectiva, com 7 anos de se guimento, em uma amostra estratificada sistemática de 335 trabalhadores da refinaria. O diag nóstico de hipertensão arterial foi feito com base nas medidas da pressão arterial obtidas nos exames médicos periódicos. A partir dos resultados do teste CAGE, aplicado ao início do período de observação, estabeleceram-se três grupos de indivíduos: não-bebedores ( $\mathrm{n}=121), C A G E-$ negativos ( $\mathrm{n}=116)$, e CAGE-positivos $(\mathrm{n}=98)$. O grupo CAGE-positivo apresentou maior risco relativo e atribuível de desenvolver hipertensão arterial do que o grupo CAGE-negativo $(R R=2,58 ; R A=24,95 \%$ pessoas-ano $)$ e que o grupo não-bebedor $(R R=2,06 ; R A=20,97 \%$ pessoas-ano). As frações atribuíveis foram $61 \%$ e $51 \%$, respectivamente, para as mesmas com parações. A padronização de taxas pela idade e hábito de fumar não modificaram substancial mente estes resultados. O alcoolismo é um importante fator de risco para o desenvolvimento de hipertensão arterial.

Nas últimas décadas, o consumo de álcool em níveis elevados vem sendo apontado como fator de risco para um número crescente de doenças (1). Dentre estas doenças, as doenças cardiovas-

\footnotetext{
Universidade Federal da Bahia, Centro de Estudos e Terapia do Abuso de Drogas, e Universidade Estadual de Feira de Santana, Departamento de Saúde. Correspondência e pedidos de separatas devem ser enviados a este autor no seguinte endereço: Centro de Estudos e Terapia do Abuso de Drogas, Rua Pedro Lessa 123, Canela, CEP 40110050, Salvador, BA, Brasil.

2 Universidade Federal da Bahia, Departamento de Medicina Preventiva, e Conselho Nacional de Desenvolvimento Científico e Tecnológico (CNPq).

3 Universidade Federal da Bahia, Departamento de Medicina Preventiva.

4 Universidade Federal da Bahia
}

culares assumem um papel de maior destaque, em particular os acidentes vasculares cerebrais (AVC) e a hipertensão arterial, sendo que esta última é reconhecida como um dos principais fatores de risco na determinação de AVC isquêmico ou hemorrágico $(2,3)$.

Algumas questões a respeito do papel desempenhado pelo padrão de ingesta alcoólica sobre a incidência de hipertensão arterial ainda não estão totalmente estabelecidas. A maior parte dos estudos aponta para a existência de uma relação linear, direta, entre o consumo de álcool e a elevação da pressão arterial (PA) (4-6). Entretanto, alguns autores apontam para a possi- bilidade de que esta relação seja do tipo "J", com o beber moderado e leve exercendo um efeito protetor (7), ou, ainda, para a existência de um limiar, abaixo do qual a ingesta etílica não exerce qualquer efeito $(8,9)$.

A maior parte dos estudos sobre associação entre consumo de álcool e hipertensão arterial foi realizada em população geral. Os raros estudos realizados em populações de trabalhadores apontam para a mesma direção, ou seja, situam o alcoolismo como importante fator de risco para hipertensão $(10,11)$. A síndrome de dependência do álcool (DAS) é muito mais freqüente em trabalhadores do que na população 
geral. A prevalência da síndrome foi de $22 \%$ entre trabalhadores químicos na Oceania (12), de $32 \%$ entre mineiros sul-africanos (13) e de $35 \%$ entre petroleiros de plataformas marítimas no Mar do Norte (14). Em um estudo realizado em população urbana de três capitais brasileiras, por outro lado, a prevalência de DAS variou de 8 a $9 \%$ (15). Portanto, é importante estudar a relação entre alcoolismo e hipertensão arterial em ambiente ocupacional. Assim, o objetivo do presente trabalho foi avaliar a associação entre alcoolismo e hipertensão arterial entre trabalhadores de uma refinaria de petróleo.

\section{MATERIAIS E MÉTODOS}

O presente artigo analisa a associação entre alcoolismo e hipertensão arterial. Os dados aqui apresentados fazem parte de um estudo mais amplo, que investiga a relação entre alcoolismo e um grupo de doenças (16) em uma coorte retrospectiva, dinâmica, de trabalhadores da Refinaria Landulpho Alves, Mataripe (RLAM). Esta refinaria de petróleo possui 1913 funcionários e está localizada na região Nordeste do Brasil. Foram utilizados dados secundários, coletados dos prontuários médicos do serviço de saúde ocupacional da refinaria no mês de agosto de 1993. O acompanhamento refere-se ao período de 1986 até 1993 . O período máximo de acompanhamento foi de 7 anos (16). A exposição foi definida, ao início do acompanhamento, através de uma triagem para alcoolismo que abrangeu a totalidade dos trabalhadores da refinaria. Durante a realização dos exames periódicos anuais, de 1986 a 1988, os médicos da empresa fizeram uma única aplicação do teste CAGE a cada um dos indivíduos que admitiam fazer uso de bebida alcoólica. Ao final de 1988, a quase totalidade dos funcionários da refinaria foi avaliada quanto ao seu padrão de ingesta etílica (17). Portanto, o início da coorte se deu neste período, de 1986 a 1988, e a entrada, na amostra, de cada indivíduo estudado, ocorreu após a avaliação do padrão de consumo alcoólico de cada trabalhador.
O teste CAGE é um questionário padronizado, composto por quatro perguntas. Sua denominação é uma acrossemia derivada das iniciais das palavras-chave de cada pergunta do original em inglês: 1) alguma vez o senhor sentiu que deveria diminuir ou parar de beber? (Cut down); 2) as pessoas o aborrecem porque criticam seu modo de beber? (Annoyed by criticism); 3) o senhor se sente culpado pela maneira como costuma beber? (Guilty about drinking); 4) o senhor costuma beber pela manhã para diminuir o nervosismo e a ressaca? (Eye-opener drinks). $\mathrm{O}$ teste CAGE visa a detecção do chamado bebedor-problema, suspeito de ser dependente de álcool. Tendo como ponto de corte duas respostas positivas às perguntas do teste, a sensibilidade do CAGE em populações hospitalares fica em torno de $88 \%$ e a especificidade, em torno de $83 \%$. Sendo de fácil aplicação, o CAGE dispensa treinamento para sua utilização (18).

Do total dos 1913 trabalhadores da refinaria, foram excluídos 54 indivíduos dos quais não se dispunha de informações adequadas sobre o padrão de consumo alcoólico, além de 109 indivíduos do sexo feminino. Restaram, portanto, 1750 trabalhadores que se constituíram na população onde se realizou o plano amostral. A partir dos dados coletados nesta triagem, foi identificado um total de 154 bebedores CAGE-positivos na refinaria, considerados bebedoresproblema (fração amostral $n / N=1,0$ ). Foram, então, identificadas amostras aleatórias, sistemáticas, estratificadas, de não-bebedores $(n=150 ; N=247$; fração amostral $n / N=0,627$ ) e de bebedores CAGE-negativos, considerados bebedores moderados $(n=150 ; N=1349$; fração amostral $n / N=0,111)$. O procedimento de amostragem sistemática e estratificada foi escolhido pela sua facilidade de execução, além de permitir a seleção de componentes para dois outros grupos em número comparável ao do grupo dos CAGE-positivos. A descrição da amostra segunda a distribuição por idade, tempo de serviço, setor de trabalho e regime de trabalho foi feita a partir de dados fornecidos pelo serviço pessoal da empresa.
O acompanhamento com vistas à determinação da incidência de hipertensão arterial foi feito através dos prontuários do serviço médico da indústria. Foram localizados 125 prontuários do grupo dos não-bebedores, 119 dos CAGE-negativos e 111 dos CAGE-positivos. Foram excluídos todos os casos prevalentes de hipertensão arterial, antes do início do acompanhamento. O número de trabalhadores sem hipertensão arterial ao início da coorte foi de 98 entre CAGEpositivos, 116 entre CAGE-negativos e 121 entre não-bebedores.

Anualmente, durante a realização dos exames periódicos, cada trabalhador teve os níveis pressóricos determinados. O serviço médico da refinaria reconhece o diagnóstico de hipertensão arterial quando os níveis pressóricos excedem os valores de $140 \times 90 \mathrm{mmHg}$. Todo trabalhador que recebeu o diagnóstico da hipertensão arterial pela primeira vez após a aplicação do teste CAGE foi considerado caso incidente.

As incidências de hipertensão em cada um dos três grupos foram calculadas pelo método da densidade de incidência (pessoas-ano) que é, na verdade, uma taxa média que mede a velocidade, a força, do adoecimento naquele grupo específico. O denominador desta taxa de incidência constitui-se do somatório dos tempos individuais de observação, iniciando a contagem a partir da aplicação do teste CAGE e finalizando no adoecimento, morte, aposentadoria, demissão ou ao final dos 7 anos do estudo. O numerador, por outro lado, constitui-se do somatório do número de casos novos de hipertensão arterial, ocorridos após a aplicação do teste CAGE em cada grupo específico. Esta forma de cálculo vem sendo utilizada em coortes ocupacionais sob a argumentação de que diminui o viés introduzido pelas perdas de acompanhamento (19).

O tempo médio de acompanhamento, em anos, foi de 5,3 para os CAGE-positivos, de 5,44 para os CAGE-negativos e de 5,05 para os nãobebedores. Foram, então, calculados risco relativo (RR), risco atribuível (RA) e fração atribuível (FA) $(20,21)$ corres- 
pondentes às comparações entre os CAGE-positivos, considerados expostos, com os CAGE-negativos e os nãobebedores. Avaliou-se o efeito das perdas de acompanhamento sobre o risco pressupondo duas situações extremas: 1) que todas as perdas desenvolveram hipertensão ao final do estudo; 2) que nenhuma das perdas desenvolveu hipertensão arterial. Foram comparados os riscos calculados a partir das três situações (mensuração efetiva e duas situações extremas) para verificar em que medida o risco efetivamente mensurado era aceitável (19). A padronização de taxas foi feita pelo método indireto (20), tomando-se sempre a composição do grupo não-exposto (CAGE-negativo ou não-bebedor) como populaçãopadrão. A análise dos dados foi feita pelo programa SPSS/PC (22).

\section{RESULTADOS}

Dos 355 trabalhadores com prontuários disponíveis, 23 tinham nos seus prontuários o diagnóstico clínico de alcoolismo anterior à aplicação do teste CAGE: seis entre os não-bebedores, três entre os CAGE-negativos e 14 entre os CAGE-positivos. Por outro lado, o exame dos prontuários dos trabalhadores, existentes no serviço médico da empresa, revelou que entre os não-bebedores havia quatro indivíduos com o diagnóstico anterior de hipertensão. Entre os CAGE-negativos, havia três trabalhadores hipertensos e, entre os CAGE-positivos, 13. Estes indivíduos foram considerados casos prevalentes de hipertensão e foram excluídos da coorte.

A tabela 1 mostra que a maior proporção dos CAGE-positivos (62\%) encontrava-se no estrato de 31 a 40 anos e que os estratos entre 21 e 30 anos e de mais de 51 anos tinham as menores proporções, com magnitude semelhante $(8 \%)$. Esta última proporção é pelo menos três vezes menor do que aquela observada entre os CAGEnegativos e entre os não-bebedores de 21 a 30 anos. Também foram identificadas diferenças substanciais entre os três grupos, em outros estratos etários,

TABELA 1. Ingesta de álcool segundo idade ao início da coorte de trabalhadores da refinaria de petróleo Landulpho Alves, Mataripe (Bahia), Brasil, 1986 a $1993^{\mathrm{a}}$

\begin{tabular}{|c|c|c|c|c|c|c|c|c|c|c|}
\hline \multirow{3}{*}{$\begin{array}{c}\text { Padrão de } \\
\text { ingesta alcoólica }\end{array}$} & \multicolumn{10}{|c|}{ Idade (anos) } \\
\hline & \multicolumn{2}{|c|}{$21-30$} & \multicolumn{2}{|c|}{$31-40$} & \multicolumn{2}{|c|}{$41-50$} & \multicolumn{2}{|c|}{$\geq 51$} & \multicolumn{2}{|c|}{ Total } \\
\hline & $n$ & $\%$ & $n$ & $\%$ & $n$ & $\%$ & $n$ & $\%$ & $n$ & $\%$ \\
\hline Não-bebedor & 29 & 24 & 48 & 40 & 27 & 22 & 16 & 13 & 120 & 100 \\
\hline CAGE-negativo & 27 & 24 & 52 & 46 & 22 & 19 & 13 & 11 & 114 & 100 \\
\hline CAGE-positivo & 8 & 8 & 61 & 62 & 21 & 21 & 8 & 8 & 98 & 100 \\
\hline
\end{tabular}

algnorados $=3 ; \chi^{2}=16,3 ; P=0,012$.

TABELA 2. Ingesta de álcool segundo regime de trabalho ao início da coorte de trabalhadores da refinaria de petróleo Landulpho Alves, Mataripe (Bahia), Brasil, 1986 a 1993a

\begin{tabular}{|c|c|c|c|c|c|c|}
\hline \multirow[b]{3}{*}{ Padrão de ingesta alcoólica } & \multicolumn{6}{|c|}{ Regime de trabalho } \\
\hline & \multicolumn{2}{|c|}{ Administrativo } & \multicolumn{2}{|c|}{$\begin{array}{l}\text { Turno de trabalho } \\
\text { alternante }\end{array}$} & \multicolumn{2}{|c|}{ Total } \\
\hline & $n$ & $\%$ & $n$ & $\%$ & $n$ & $\%$ \\
\hline Não-bebedor & 64 & 55 & 53 & 45 & 117 & 100 \\
\hline CAGE-negativo & 48 & 41 & 68 & 59 & 116 & 100 \\
\hline CAGE-positivo & 41 & 42 & 57 & 58 & 98 & 100 \\
\hline
\end{tabular}

Ignorados $=4 ; \chi^{2}=5,4 ; g \mid=4 ; P=0,25$.

atingindo elevada significância estatística $\left(\chi^{2}=16,3 ; P=0,012\right)$.

A distribuição segundo regime de trabalho (tabela 2 ) revelou que o regime administrativo era predominante entre os não-bebedores (55\%), ao passo que o regime de trabalho de turno alternante (TTA), ao início do acompanhamento desta coorte, foi mais freqüente entre os CAGE-positivos (58\%) e os CAGE-negativos (59\%). No regime administrativo, o trabalho, naquela refinaria, é realizado durante o dia de segunda à sexta-feira, de 7 às 17 horas. $\mathrm{O}$ regime de TTA predomina entre os trabalhadores da produção da refinaria. Estas diferenças não alcançaram nível elevado de significância estatística $\left(\chi^{2}=\right.$ $5,4 ; P=0,25)$.

A distribuição dos três padrões de ingesta segundo tempo de serviço evidenciou que os maiores contingentes de trabalhadores trabalhavam na empresa há 16 anos ou menos, seja entre os CAGE-positivos (72\%), entre os CAGE-negativos (74\%), ou ainda entre os não-bebedores (65\%). Ademais, as diferenças encontradas não foram sig- nificativas do ponto de vista estatístico $\left(\chi^{2}=12,01 ; P=0,284\right)$.

Observou-se que, nos três grupos, predominavam os trabalhadores da área de operação, onde são realizadas as atividades de refino de petróleo (tabela 3). Em seguida, vinham os trabalhadores das áreas de manutenção, administração e segurança, respectivamente $\left(\chi^{2}=12,3 ; P=0,056\right)$.

A freqüência do hábito de fumar tendeu a crescer com a mudança do padrão de ingesta alcoólica: entre os não bebedores, 14\% eram fumantes; entre os CAGE-negativos, 30\%; e entre os CAGE-positivos, $45 \%$ eram fumantes $\left(\chi^{2}=28,1 ; P=0,0001\right)$ (tabela 4$)$.

A tabela 5 mostra que a proporção de prontuários localizados foi menor no grupo dos CAGE-positivos (111/ $154=72 \%$ ) e maior entre os não-bebedores $(125 / 150=83 \%)$. As perdas de acompanhamento no decorrer dos 7 anos de seguimento da coorte foram menores no grupo dos CAGE-positivos $(28 \%)$ do que no grupo dos CAGEnegativos (35\%) e dos não-bebedores (37\%). Entretanto, estas diferenças não 
TABELA 3. Ingesta alcoólica segundo as quatro grandes áreas administrativas ao início de uma coorte de trabalhadores da refinaria de petróleo Landulpho Alves, Mataripe (Bahia), Brasil, 1986 a $1993^{a}$

\begin{tabular}{|c|c|c|c|c|c|c|c|c|c|c|}
\hline \multirow{3}{*}{$\begin{array}{l}\text { Padrão de ingesta } \\
\text { alcoólica }\end{array}$} & \multicolumn{10}{|c|}{ Áreas administrativas } \\
\hline & \multicolumn{2}{|c|}{ Administração } & \multicolumn{2}{|c|}{ Segurança } & \multicolumn{2}{|c|}{ Operação } & \multicolumn{2}{|c|}{ Manutenção } & \multicolumn{2}{|c|}{ Total } \\
\hline & $n$ & $\%$ & $n$ & $\%$ & $n$ & $\%$ & $n$ & $\%$ & $n$ & $\%$ \\
\hline Não-bebedor & 26 & 22 & 11 & 9 & 52 & 44 & 29 & 25 & 118 & 100 \\
\hline CAGE-negativo & 15 & 13 & 7 & 6 & 66 & 59 & 24 & 21 & 112 & 100 \\
\hline CAGE-positivo & 16 & 16 & 1 & 1 & 57 & 58 & 24 & 24 & 98 & 100 \\
\hline
\end{tabular}

algnorados $=7 ; \chi^{2}=12,3 ; P=0,056$.

TABELA 4. Distribuição do hábito de fumar segundo padrão de ingesta alcoólica da coorte de trabalhadores da refinaria de petróleo Landulpho Alves, Mataripe (Bahia), Brasil, 1986 a $1993^{\mathrm{a}}$

\begin{tabular}{|c|c|c|c|c|c|c|}
\hline \multirow[b]{3}{*}{ Padrão de ingesta alcoólica } & \multicolumn{6}{|c|}{ Hábito de fumar } \\
\hline & \multicolumn{2}{|c|}{ Fumante } & \multicolumn{2}{|c|}{ Não-fumante } & \multicolumn{2}{|c|}{ Total } \\
\hline & $n$ & $\%$ & $n$ & $\%$ & $n$ & $\%$ \\
\hline Não-bebedor & 17 & 14 & 104 & 86 & 121 & 100 \\
\hline CAGE-negativo & 34 & 30 & 79 & 70 & 116 & 100 \\
\hline CAGE-positivo & 43 & 45 & 53 & 55 & 96 & 100 \\
\hline
\end{tabular}

agnorados $=2 ; \chi^{2}=28,14 ; g \mid=4 ; P<0,0001$.

TABELA 5. Características da composição dos grupos e respectivas perdas de acompanhamento da coorte, refinaria de petróleo Landulpho Alves, Mataripe (Bahia), Brasil, 1986 a 1993

\begin{tabular}{|c|c|c|c|c|c|}
\hline \multirow{2}{*}{$\begin{array}{c}\text { Padrão de } \\
\text { ingesta alcoólica }\end{array}$} & \multirow[b]{2}{*}{$N$ inicial } & \multirow{2}{*}{$\begin{array}{l}\text { Prontuários } \\
\text { localizados }\end{array}$} & \multirow{2}{*}{$\begin{array}{c}\text { Casos } \\
\text { prevalentes }\end{array}$} & \multicolumn{2}{|c|}{$\begin{array}{c}\text { Perdas no decorrer } \\
\text { da coorte }\end{array}$} \\
\hline & & & & $n$ & $\%$ \\
\hline Não-bebedor & 150 & 125 & 4 & 45 & 37 \\
\hline CAGE-negativo & 150 & 119 & 3 & 41 & 35 \\
\hline CAGE-positivo & 154 & 111 & 13 & 27 & 28 \\
\hline
\end{tabular}

${ }^{a} P=0,13$.

foram estatisticamente significativas $(P=0,13)$.

A incidência de hipertensão arterial (tabela 6) foi maior entre os CAGEpositivos $(40,78 \%$ pessoas-ano $)$ e menor entre os CAGE-negativos (15,82\% pessoas-ano), sendo esta apenas um pouco menor do que a incidência encontrada entre os não-bebedores $(19,8 \%$ pessoas-ano). A tabela 7 mostra que o grupo dos indivíduos CAGE-positivos apresentou maior risco relativo e atribuível de desenvolver hiperten-
A tabela 8 mostra a comparação entre os RR efetivamente mensurados $\mathrm{e}$ os riscos hipoteticamente encontrados em situações extremas. Assim, calculase o risco supondo acompanhar todas as perdas até o final do estudo, elas mantendo-se sadias ou adoecendo ao fim do acompanhamento. Verifica-se, pela análise dos intervalos de confiança, que não existem diferenças estatísticas entre as três formas de calcular o RR ao nível de 5\% de probabilidade.

\section{DISCUSSÃO}

Os resultados do presente estudo sugerem que o alcoolismo é um fator de risco para o desenvolvimento de pressão arterial. A padronização de taxas por idade e hábito de fumar e as perdas de acompanhamento não modificaram substancialmente os resultados.

Na relação entre consumo de álcool e qualquer doença a ele associada, deve-se considerar os critérios de definição da variável independente, da exposição. Neste estudo, a exposição foi definida pelo teste CAGE, que visa detectar bebedores-problema, ou seja, aqueles indivíduos suspeitos de serem dependentes de álcool (18). O teste foi aplicado em ambiente ocupacional, o que reforça o temor do estigma do alcoolismo, podendo interferir grandemente nas respostas dos entrevistados. Constatou-se a existência de um indivíduo com diagnóstico anterior de alcoolismo entre os não-bebedores, e de sete indivíduos entre os CAGE-negativos. Este tipo de ocorrência é extremamente comum na literatura científica sobre o tema. É fato conhecido que algumas doenças associadas ao alcoolismo influenciam a mudança no padrão de ingesta, sendo a mudança mais comum a que vai do "beber pesado" para o "não beber" (23). Este tipo de erro de classificação, que coloca um certo número de expostos entre os não-expostos, na vigência de relação causal comprovada, subestima as relações de associação, em particular o RA (24).

A descrição dos três grupos de ingesta alcoólica ao início da coorte visou principalmente analisar a sua comparabilidade. Assim, verificou-se que estes 
TABELA 6. Incidência de hipertensão arterial em uma coorte de trabalhadores segundo padrão de ingesta alcoólica, refinaria de petróleo Landulpho Alves, Mataripe (Bahia), Brasil, 1986 a 1993

\begin{tabular}{ccccc}
\hline Padrão de ingesta alcoólica & $N$ inicial & $n$ doença & Pessoas-ano & Incidência (\% P-A) \\
\hline Não-bebedor & 121 & 12 & 606 & 19,80 \\
CAGE-negativo & 116 & 10 & 632 & 15,82 \\
CAGE-positivo & 98 & 21 & 515 & 40,78 \\
\hline
\end{tabular}

grupos não eram comparáveis em relação à idade e ao hábito de fumar, o que não é a situação ideal para uma coorte. Estas co-variáveis podem, inclusive, estar relacionadas com alcoolismo; entretanto, o fato de que a padronização de taxas por idade e por hábito de fumar não alterou a magnitude das medidas de associação não é fato incomum na literatura (25).

Um dos problemas fundamentais desta coorte é o número bastante elevado de acompanhamento. Embora, ao final do estudo, valores próximos de perdas nos três grupos sejam atingidos, deve ser notado que a velocidade foi diferente em cada um deles. As perdas foram mais precoces entre os bebedores-problema do que nos outros grupos. Assim, pode-se pensar que estas perdas relacionavam-se com a exposição. Não pode ser afirmado que as perdas também se relacionavam com o efeito em questão, embora esta possibilidade também não possa ser descartada a partir dos dados. É importante lembrar que, sempre que as perdas de acompanhamento se relacionarem, seja com a exposição, seja com o efeito, elas estarão introduzindo um viés de seleção (20).

Nas coortes ocupacionais, especialmente nas retrospectivas, o problema das perdas é mais freqüente do que na população em geral. Estas populações têm uma dinâmica própria, com rotatividade maior ou menor, a depender do estudo. Esta rotatividade é dependente, entre outros, das demissões ou das aposentadorias. Já está fartamente documentado na literatura que estes fenômenos ocorrem com mais freqüência entre dependentes de álcool do que entre outros trabalhadores (11).

Existem outras formas de calcular o risco na vigência de perdas elevadas. Uma delas, preconizada pelo National Institute of Occupational Safety and Health (NIOSH) $(19,26)$, supõe que as perdas chegaram até o final do estudo e, até aquele momento, mantiveram-se sadias. A terceira forma, que supõe o adoecimento ao final do estudo, é menos utilizada.

O que o NIOSH sugere é, na verdade, ampliar o denominador (pessoas-ano) do cálculo das incidências, mantendose o numerador efetivamente mensu- rado (número de doentes). Esta forma de cálculo subestima o risco, o que também acontece com a terceira forma de cálculo (19). O que esta instituição pretende é que, se ainda assim for encontrado risco maior do que a unidade, é porque ele efetivamente existe na população. A proposta do NIOSH vem sendo criticada pelo fato de ampliar, em muito, na análise de dados, o efeito trabalhador sadio, que pode ser observado pelo fato de que, em coortes ocupacionais, as taxas de morbidade e mortalidade são geralmente menores do que na população geral. Este efeito depende, dentre outros fatos, da seleção e da manutenção dos sadios na integração da força ativa de trabalho (26).

$\mathrm{O}$ presente estudo não encontrou diferenças significativas do ponto de vista estatístico entre as medidas de associação avaliadas, além de verificar que as outras formas de calcular o risco tiveram magnitude realmente menor. Estas evidências sugerem que o risco efetivamente mensurado a partir dos dados desta coorte deve ser tomado como uma medida aceitável.

Como os dados utilizados pelo presente estudo são secundários, não houve um controle rigoroso de sua qualidade. Isto amplia a possibilidade de perda de alguns casos de hipertensão nesta coorte. $O$ principal problema para a comparação entre estes dados e os dados de outros estudos é o fato de que a maior parte dos pesquisadores trabalhou com indicadores de consumo do tipo quantidade e freqüência, que são tão permeáveis a viés de informação quanto os questionários padro-

TABELA 7. Risco relativo, razão de densidade de incidência padronizada, risco atribuível e fração atribuível de hipertensão arterial em uma coorte de trabalhadores segundo o padrão de ingesta alcoólica, refinaria de petróleo Landulpho Alves, Mataripe (Bahia), Brasil, 1986 a 1993

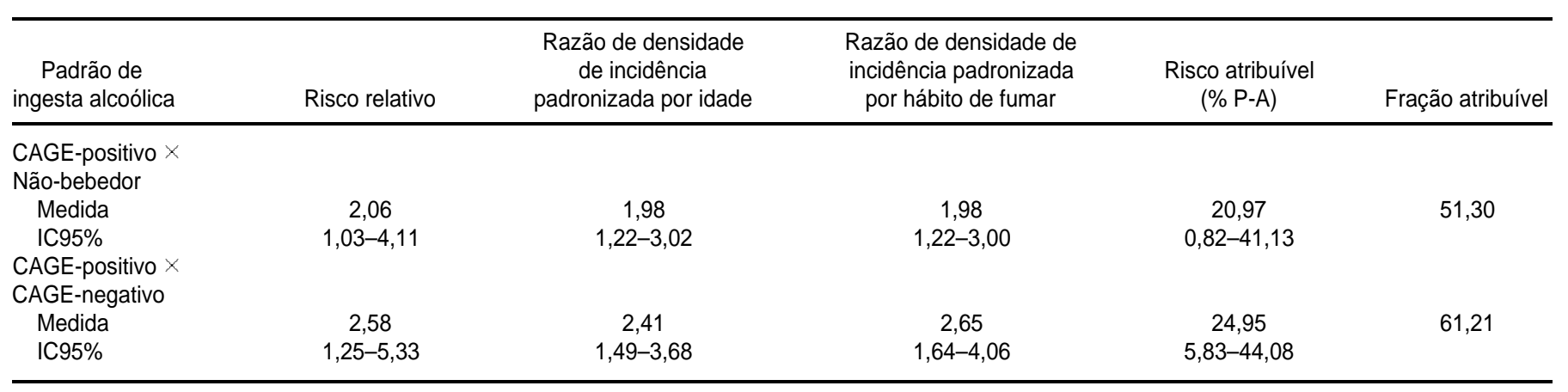


TABELA 8. Avaliação da influência das perdas de acompanhamento sobre o cálculo do risco relativo de hipertensão arterial segundo o padrão de ingesta alcoólica, refinaria de petróleo Landulpho Alves, Mataripe (Bahia), Brasil, 1986 a 1993

\begin{tabular}{cccc}
\hline Padrão de ingesta alcoólica & Mensurado & $\begin{array}{c}\text { Perdas } \\
\text { supostas sadias }\end{array}$ & $\begin{array}{c}\text { Perdas } \\
\text { supostas doentes }\end{array}$ \\
\hline CAGE-positivo & & & \\
Não-bebedor & & & 1,18 \\
$\quad$ Risco relativo & 2,06 & 1,85 & $0,87-1,58$ \\
IC95\% & $1,03-4,11$ & $0,92-3,73$ & \\
CAGE-positivo & & & 1,20 \\
CAGE-negativo & 2,58 & 2,28 & $0,90-1,62$ \\
Risco relativo & $1,25-5,33$ & $1,09-4,73$ & \\
IC95\% & & & \\
\hline
\end{tabular}

nizados do tipo CAGE (27). Ainda assim, é possível afirmar que existe coerência entre os resultados desta coorte e os de diversos estudos realizados em população geral, que apontam o "beber pesado" como fator de risco para a hipertensão (2-9). Numa das poucas coortes realizadas em população de trabalhadores (11) encontrou-se um risco relativo igual a 2,3, portanto de magnitude muito próxima aos riscos observados neste estudo.

A incidência de hipertensão arterial encontrada no presente estudo para o grupo dos CAGE-negativos e nos nãobebedores é coerente com os trabalhos que apontam para a existência de um limiar abaixo do qual o consumo de álcool não teria nenhum efeito $(3,8)$. Al- guns autores (4-6) referem a existência de uma relação linear entre consumo de álcool e incidência de hipertensão. Outros autores $(23,28)$ argumentam que o não beber e o beber moderado podem estar associados a uma série de outros hábitos de vida mais saudáveis e que podem contribuir para este efeito protetor.

Diversos mecanismos vêm sendo aventados para explicar a plausibilidade biológica da associação entre hipertensão e alcoolismo. Além do estresse, tem sido proposta a elevação das catecolaminas como explicação (29). Sabe-se, entretanto, que o álcool interfere no sistema renina-angiotensina (2) e que bebedores pesados têm baixa absorção de cálcio (30), podendo ser este um mecanismo adicional para o desenvolvimento da hipertensão arterial.

Uma última questão, talvez a principal do presente estudo, é a magnitude da fração atribuível. A fração atribuível permite observar o que ocorreria em termos de redução das taxas de hipertensão entre os expostos, bebedoresproblema, se houvesse uma mudança no padrão de ingesta. Reafirma-se que esta medida foi calculada a partir de um indicador de exposição (teste CAGE) que se revelou pouco sensível, o que pode subestimar a fração atribuível (24). Ainda assim, esta medida fornece subsídios para o planejamento de ações de saúde específicas, do tipo programas de tratamento de alcoolismo para empregados de grandes indústrias como a RLAM. Programas deste tipo são necessários, considerando-se que a hipertensão arterial é um importante fator de risco para AVC, que é a primeira causa de óbito específica no Brasil (31).

Agradecimentos. A realização deste trabalho não seria possível sem o valioso apoio dos seguintes profissionais da refinaria: Dr. José F. Gidi, então chefe do Serviço de Saúde Ocupacional; Dr. Roberto Charles Góes, médico do trabalho; Sra. Elisabeth M. Martins, assistente social; e Sr. Florivaldo, arquivista. Especial agradecimento para o quarto e último revisor deste manuscrito, por suas valiosas sugestões.

\section{REFERÊNCIAS}

1. Brody JA, Mills GS. On considering alcohol as risk factor in specific diseases. Am J Epidemiol 1978;107(6):462-466.

2. Clark L. Alcohol use and hypertension: clinical considerations and implications. Postgrad Med 1984;75(8):273-276.

3. Ueshima H, Ohsaka T, Tatara K, Asakura S. Alcohol consumption, blood pressure and stroke mortality in Japan. J Hypertens 1984; 2(Suppl 3):191-195.

4. Goldberg RJ, Burchfiel CM, Reed DM, Wergowske $G$, Chiu D. A prospective study of the health effects of alcohol consumption in middle-aged and elderly men: the Honolulu health program. Circulation 1994;89(2): 651-659.

5. Kozarevic D, Racic Z, Gordon T, Kaelber C, McGee D, Zukel W. The Yugoslavian cardio- vascular disease study: drinking habits and associated characteristics. Am J Epidemiol 1982;116(2):287-301.

6. Wakabayashi K, Nakamura K, Kono S, Shinchi K, Imanishi K. Alcohol consumption and blood pressure: an extended study of selfdefense officials in Japan. Int J Epidemiol 1994;23(2):307-311.

7. Jackson R, Stewart A, Beaglehole R, Scrag R. Alcohol consumption and blood pressure. Am J Epidemiol 1985;122(6):1037-1344.

8. Sorel JE. Alcohol and blood pressure. Epidemiology 1994;5(6):567-569.

9. Van Leer EM, Seidel JC, Kromhout D. Differences in the association between alcohol consumption and blood pressure by age, gender, and smoking. Epidemiology 1994;5(6): 576-582.
10. Dyer AR, Stamler J, Paul O, Berkson DM, Lepper MH, McKean H, et al. Alcohol consumption, cardiovascular risk factors and mortality in two Chicago epidemiologic studies. Circulation 1977;56(6):1067-1074.

11. Pell S, D'Alonzo CA. The prevalence of chronic heart disease among problem drinkers. Arch Environ Health 1968;16(5): 679-684.

12. Webb GR, Redman S, Hennrikus D, Rostas D, Sansonfisher W. The prevalence and sociodemographic correlates of high-risk and problem drinking at an industrial worksite. $\mathrm{Br} \mathrm{J}$ Addict 1990;85(4):495-507.

13. Lewis KO, Paton A. ABC of alcohol: tools of detection. Br Med J 1981;283(6305):1531-1532.

14. Aiken GJ \& McCance C. Alcohol consumption in offshore oil rig workers. Br J Addict 1982; 77(3):305-310. 
15. Almeida Filho N, Mari JJ, Coutinho E, França JF, Fernandes JG, Andreoli SB, et al. Estudo multicêntrico de morbidade psiquiátrica em áreas urbanas brasileiras (Brasília, São Paulo, Porto Alegre). Revista ABP-APAL 1992;14(3): 93-104.

16. Lima CTS. Alcoolismo e doenças associadas. Um estudo de coorte em trabalhadores de uma refinaria de petróleo [dissertação de mestrado]. Salvador: Programa de Saúde Comunitária da Universidade Federal da Bahia; 1995.

17. Gidi JF, Torres PB, Menezes JM, Santos EAJ, Góes RCS. Utilização da dosagem de gamaglutamil-transferase associada ao teste CAGE no diagnóstico do alcoolismo na empresa. Em: Sétimo Congresso da Associação Brasileira de Estudos do Álcool e Outras Drogas; 1987; Gramado, RS, Brasil.

18. Masur J, Monteiro MG. Validation of the "CAGE" alcoholism screening test in a Brazilian psychiatric inpatient hospital setting. Braz J Med Biol Res 1983;16(3):215-218.

19. Vena JE, Sultz HA, Carlo GL, Fielder RC, Barnes RE. Sources of bias in retrospective co- hort mortality studies: a note on treatment of subjects lost to follow-up. J Occup Med 1987; 29(3):256-261.

20. Rothman KJ. Modern epidemiology. Boston: Little, Brown and Company; 1986.

21. Hennekens $\mathrm{CH}$, Buring JE. Epidemiology in medicine. Boston: Little, Brown and Company; 1987.

22. Norussis MJ. SPSS + V2.0 Base manual. Chicago: SPSS Inc; 1988.

23. Beilin LJ. Alcohol, hypertension and cardiovascular disease [editorial]. J Hypertens 1995; 13(9):939-942.

24. Wacholder S, Benichou J, Heineman EF, Hartge P, Hoover RN. Attributable risk: advantages of a broad definition of exposure. Am J Epidemiol 1994;140(4):303-309.

25. Ahlawat SK, Siwach SB. Alcohol and coronary artery disease. Int J Cardiol 1994;44(2): 157-162.

26. Checkoway H, Pearce N, Crawford-Brown D. Research methods in occupational epidemiology: Monographs in epidemiology and biostatistics. Vol 13. New York: Oxford University Press; 1989.
27. Coutinho ESF. Alcoolismo e problemas relacionados; dificuldades na implementação de estudos de prevalência. Cad Saude Publica 1992;8(1):22-29.

28. Shaper AG. Alcohol intake and mortality [carta]. Br Med J 1994;308(6928):598.

29. Kaysen G, Noth RH. Efeitos do álcool sobre a pressão arterial e os eletrólitos. Clinicas Medicas America do Norte 1984;68(1):235-261.

30. Criqui MH. The roles of alcohol in the epidemiology of cardiovascular diseases. Acta Med Scand 1987:717(suppl):73-85.

31. Possas C. Epidemiologia e sociedade: heterogeneidade estrutural e saúde no Brasil. São Paulo: Editora Hucitec; 1989.

Manuscrito recebido em 26 de junho de 1996. Aceito em versão revisada em 6 de fevereiro de 1997.

ABSTRACT The role of alcohol ingestion in the incidence of arterial hypertension has not been completely established. In addition, there are few studies addressing this point in relation to populations of workers. The objective of this study was to evaluate the association between alcoholism and arterial hypertension among workers in an oil refinery in Mataripe, Bahia, Brazil, from 1986 to 1993. We designed a retrospective cohort study with a 7-year follow-up in a stratified systematic sample of 335 workers from the refinery. Arterial hypertension was diagnosed based on blood pressure measurements done during routine medical examinations. At the beginning of follow-up, three groups were defined using the CAGE test of alcohol dependency: nondrinkers $(n=121)$, CAGE-negative workers $(n=116)$, and CAGE-positive workers $(n=98)$. In comparison with the CAGE-negative group, the CAGE-positive group had both greater relative risk and greater attributable risk for developing arterial hypertension $(\mathrm{RR}=2.58 ; \mathrm{AR}=24.95$ per 1000 person-years). The CAGE-positive group also had greater risks compared to nondrinkers $(\mathrm{RR}=2.06$; $\mathrm{AR}=20.97$ per 1000 person-years $)$. The attributable fractions for the same two comparisons of groups were $61 \%$ and $51 \%$, respectively. Rate standardization by age or smoking habit did not substantially change the results. Alcoholism is an important risk factor for arterial hypertension. 